\title{
Effect of Plant Population and Fertilizer Doses on Yield Attributes, Yield and Economics of Summer Groundnut
}

\author{
B.D. Waghmode ${ }^{1 *}$, A.S. Kambale ${ }^{1}$, V.C. Navhale ${ }^{1}$, P.D. Chendge ${ }^{1}$ and U.V. Mahadkar ${ }^{2}$ \\ ${ }^{1}$ Agricultural Research Station, Shirgaon, Dist. Ratnagiri - 415629 (MS), India \\ ${ }^{2}$ Department of Agronomy, Collage of Agriculture, Dapoli-415 712, Maharashtra, India \\ *Corresponding author
}

\section{A B S T R A C T}

\section{Keywords}

Plant population,

Levels of fertilizer,

Economics, Dry pod yield.

Article Info

Accepted:

20 September 2017

Available Online:

10 November 2017
A field experiment was conducted at Agricultural Research Station, Shirgaon, Ratnagiri (MS) on the lateritic soil during Rabi 2013-14 to 2015-16 to study effect of plant population and fertilizer doses on growth and yield of summer groundnut. The treatment comprises four main plot treatments of planting geometry and three sub-plot treatments of levels of fertilizer replicated three times in split plot design. Groundnut cultivar, TKG Bold sown with planting geometry $20 \times 10 \mathrm{~cm}$ (plant population @ 5 lakhs ha ${ }^{-1}$ ) with application of $125 \%$ RDF level noticed significantly highest pod, kernel and haulm yield of groundnut, which was followed by planting geometry $25 \times 10 \mathrm{~cm}$ (plant population @ 4.0 lakhs ha ${ }^{-1}$ ), 30 X $10 \mathrm{~cm}$ (plant population@3.33 lakhs ha ${ }^{-1}$ ) and 30 X $15 \mathrm{~cm}$ (plant population@2.22 lakhs ha ${ }^{-1}$ ) with same fertilizer level i.e.125\% RDF. The groundnut cultivar, TKG Bold sown with planting geometry $30 \times 15 \mathrm{~cm}$ (Plant Population of 2.22 lakhs ha $\left.{ }^{-1}\right)$ and fertilized with $125 \%$ RDF level $\left(\mathrm{P}_{1} \mathrm{~F}_{3}\right)$ recoded significantly highest number of pods plant ${ }^{-1}$ and pod weight plant ${ }^{-1}$ over rest of treatment combinations. However, the highest benefit to cost ratio was observed in planting geometry $30 \times 15 \mathrm{~cm}$ with $125 \%$ $\mathrm{RDF}$ level $\left(\mathrm{P}_{1} \mathrm{~F}_{3}\right)$.

\section{Introduction}

Groundnut (Arachis hypogaea L.) is a major oilseed crop in India. Its seed contain high quality of $45-50 \%$ edible oil, $25-30 \%$ digestible protein, $20 \%$ carbohydrates, and $5 \%$ fiber and ash which make a sustainable contribution to human nutrition (Fageria et al., 1997). Groundnut is considered as poor man's almond and also called as "King of oilseeds". It is cultivated in India over 45.97 million ha area with the production of 67.33 million tonnes (both kharif and rabi) and average productivity of $1.47 \mathrm{t} \mathrm{ha}^{-1}$ (Indiastat, 2016). In Maharashtra, groundnut cultivated (both kharif and rabi) over an area of 3.09 million ha with production of 3.34 million tonnes having productivity of $1.08 \mathrm{t} \mathrm{ha}^{-1}$ (Indiastat, 2016). The area under groundnut crop in konkan was about more than 15,000 ha with $1,550 \mathrm{~kg} \mathrm{ha}^{-1}$ productivity during 2013-14 (Anonymous, 2013).

Planting geometry is an important agronomical management practice and nonmonetary input, which has key role in increased crop production. Crop planted in appropriate geometry enhances use of natural resources as well as inputs given to the crop. Groundnut crop competes with each other 
above and below the ground. Planting geometry varies according to species and region. Similarly, appropriate fertilizer dose is also an important aspect regarding crop production. Supplying nutrients in required quantity and fulfills crop need, increases the potential of cultivar. Study of different levels of fertilizer to crop helps to standardize the fertilizer dose for getting profitable yield. Groundnut variety TKG-Bold (Trombay Konkan Groundnut-Bold') is promising, semi spreading and bold seeded variety. This variety is popular among the farmers. So therefore it is necessary to find appropriate planting geometry and fertilizer dose for getting profitable yield. Hence therefore the present investigation was undertaken.

\section{Materials and Methods}

The present investigation was conducted at Agricultural Research Station, Dr. B.S. Konkan Krishi Vidyapeeth, Shirgaon, Dist. Ratnagiri (MS) during the three successive rabi seasons from 2013-14 to 2015-16 with Groundnut cultivar Trombay Konkan Groundnut-Bold' (TG 19A). The experiment was laid out in split plot design replicated three times. The main plot treatment consists of four planting geometry viz., $\mathrm{P}_{1}$ : Plant Population@ 2.22 lakhs ha-1 $\left(30\right.$ x15 cm), $\mathrm{P}_{2}$ : Plant Population@3.33 lakhs ha ${ }^{-1}(30 \times 10$ cm), $\mathrm{P}_{3}$ : Plant Population@ @ 4.0 lakhs ha ${ }^{-1}$ (25 x $10 \mathrm{~cm}$ ) and $\mathrm{P}_{4}$ : Plant Population @ 5.0 lakhs $\mathrm{ha}^{-1}(20 \mathrm{x} 10 \mathrm{~cm})$. However, sub plot treatments consist of three levels of fertilizers viz., $\mathrm{F}_{1}: 75 \% \mathrm{RDF}, \mathrm{F}_{2}: 100 \% \mathrm{RDF}$ and $\mathrm{F}_{3}$ : $125 \%$ RDF. The soil was sandy loam in texture and acidic in reaction ( $\mathrm{pH} 5.2)$, low in available nitrogen $\left(277.2 \mathrm{~kg} \mathrm{ha}^{-1}\right)$, phosphorus (13.3 $\mathrm{kg} \mathrm{ha}^{-1}$ ) and medium in available potassium (186.5 $\left.\mathrm{kg} \mathrm{ha}^{-1}\right)$. Groundnut seed was sown at the rate of $125 \mathrm{~kg} \mathrm{ha}^{-1}$ with spacing as per treatments. In nutrient management, urea was applied as source of nitrogen while Single Super Phosphate (SSP) for phosphorus. All these treatments were imposed as per the schedule and statistical data of these variables obtained during the course of investigation were analyzed by analysis of variance method as per the procedure (Split plot design) described by Gomez and Gomez (1983) and Panse and Sukhatme (1985).

\section{Results and Discussion}

\section{Ancillary growth and yield attributes}

Pooled data presented in Table 1 indicates that, plant height, number of branches plant ${ }^{-1}$, number of pods plant ${ }^{-1}$ and pod weight plant $^{-1}$ were significantly influenced due to different planting geometry. Groundnut sown at widely spaced plant population of 2.22 lakhs ha ${ }^{-1}$ (30 $\mathrm{x} 15 \mathrm{~cm}$ ) had significantly highest growth and yield attributing characters over rest of planting geometry. This was due to more space associated with plant, which leads to more growth and yield attributes. However, plant height was significantly higher in closely spaced Plant Population of 5.0 lakhs $\mathrm{ha}^{-1}(20 \mathrm{x} 10 \mathrm{~cm})$ over rest of planting geometry. This is due to more competition among the plant for sunlight, nutrients and space. These results are in hormone with the findings of Haricharan Reddy et al., (2014), Meena (2011) and Morshed Alam et al., (2002).

There was significant difference observed for plant height, number of pods plant ${ }^{-1}$ and pod weight plant ${ }^{-1}$ of groundnut due to different levels of fertilizer treatments under study. Groundnut fertilized with $125 \%$ RDF noticed significantly highest plant height $(50.1 \mathrm{~cm})$, number of pods plant ${ }^{-1}$ (16.4) and pod weight plant $^{-1}(21.9 \mathrm{~g})$ of groundnut over rest of levels of fertilizer. However, number of branches plant ${ }^{-1}$ does not differed due to levels of fertilizer. Similar results were reported by Jakusko and Dakato (2015). 
Table.1 Plant height, number of branches, total number of pods and pod weight per plant of groundnut at harvest as influenced by different treatments under summer season (Pooled data of three years)

\begin{tabular}{|c|c|c|c|c|c|c|c|c|c|c|c|c|c|c|c|c|}
\hline \multirow[b]{2}{*}{ Treatments } & \multicolumn{4}{|c|}{ Plant height $(\mathrm{cm})$ at harvest } & \multicolumn{4}{|c|}{ No. of branches plant ${ }^{-1}$} & \multicolumn{4}{|c|}{ Total number of pods plant ${ }^{-1}$} & \multicolumn{4}{|c|}{ pod weight $\left(\mathrm{g} \mathrm{plant}^{-1}\right)$} \\
\hline & $\begin{array}{l}F_{1}: 75 \\
\% \text { RDF }\end{array}$ & $\begin{array}{c}\text { F2 : } \\
100 \% \\
\text { RDF }\end{array}$ & $\begin{array}{c}\text { F3 : } \\
125 \% \\
\text { RDF }\end{array}$ & Mean & $\begin{array}{l}F_{1}: 75 \\
\% \text { RDF }\end{array}$ & $\begin{array}{c}\text { F2 : } \\
100 \% \\
\text { RDF }\end{array}$ & $\begin{array}{c}\text { F3 : } \\
125 \% \\
\text { RDF }\end{array}$ & Mean & $\begin{array}{c}\mathbf{F}_{1}: \\
75 \% \\
\text { RDF }\end{array}$ & $\begin{array}{c}\text { F2 : } 100 \\
\% \text { RDF }\end{array}$ & $\begin{array}{c}\text { F3 : } \\
125 \% \\
\text { RDF } \\
\end{array}$ & Mean & $\begin{array}{c}\mathrm{F}_{1}: \mathbf{7 5} \\
\% \\
\text { RDF }\end{array}$ & $\begin{array}{c}\text { F2: } \\
100 \% \\
\text { RDF }\end{array}$ & $\begin{array}{c}\text { F3 : } \\
125 \% \\
\text { RDF }\end{array}$ & Mean \\
\hline$P_{1}:(30 \times 15 \mathrm{~cm})$ & 44.0 & 45.2 & 47.5 & 45.6 & 2.89 & 3.11 & 3.18 & 3.06 & 14.9 & 16.4 & 20.0 & $\begin{array}{ll}17.1 \\
\end{array}$ & 20.0 & 22.2 & 26.6 & 23.0 \\
\hline$P_{2}:(30 \times 10 \mathrm{~cm})$ & 44.5 & 45.5 & 47.5 & 45.8 & 2.87 & 2.96 & 3.11 & 2.98 & 14.7 & 16.1 & 17.9 & 16.2 & 19.8 & 20.3 & 23.8 & 21.3 \\
\hline$P_{3}:(25 \times 10 \mathrm{~cm})$ & 46.7 & 48.3 & 51.7 & 48.9 & 2.82 & 2.87 & 2.84 & 2.84 & 13.2 & 13.7 & 14.9 & 13.9 & 16.6 & 19.3 & 21.7 & 19.2 \\
\hline$P_{4}(20 \times 10 \mathrm{~cm})$ & 47.4 & 50.7 & 53.7 & 50.6 & 2.76 & 2.62 & 2.73 & 2.70 & 10.2 & 12.1 & 12.8 & 11.7 & 13.6 & 15.3 & 15.7 & 14.9 \\
\hline Mean & 45.6 & 47.4 & 50.1 & 47.7 & 2.83 & 2.89 & 2.97 & 2.90 & 13.3 & 14.6 & 16.4 & 14.7 & 17.5 & 19.3 & 21.9 & 19.6 \\
\hline Comparing the means of & \multicolumn{2}{|c|}{ S.E. \pm} & \multicolumn{2}{|c|}{$\operatorname{LSD}(0.05)$} & \multicolumn{2}{|c|}{ S.E. \pm} & \multicolumn{2}{|c|}{$\operatorname{LSD}(0.05)$} & \multicolumn{2}{|c|}{ S.E. \pm} & \multicolumn{2}{|c|}{$\operatorname{LSD}(0.05)$} & \multicolumn{2}{|c|}{ S.E. \pm} & \multicolumn{2}{|c|}{$\operatorname{LSD}(0.05)$} \\
\hline Planting Geometry $(\mathrm{P})$ & \multicolumn{2}{|c|}{$\frac{0.7}{0.5}$} & \multicolumn{2}{|c|}{2.0} & \multicolumn{2}{|c|}{0.06} & \multicolumn{2}{|c|}{0.17} & \multicolumn{2}{|c|}{0.3} & \multicolumn{2}{|c|}{0.9} & \multicolumn{2}{|c|}{0.4} & \multicolumn{2}{|c|}{1.2} \\
\hline Fertility levels (F) & \multirow{2}{*}{\multicolumn{2}{|c|}{$\frac{0.5}{1.0}$}} & \multirow{2}{*}{\multicolumn{2}{|c|}{$\frac{1.4}{2.8}$}} & \multirow{2}{*}{\multicolumn{2}{|c|}{$\begin{array}{l}0.05 \\
0.11\end{array}$}} & \multirow{2}{*}{\multicolumn{2}{|c|}{ NS }} & \multirow{2}{*}{\multicolumn{2}{|c|}{0.5}} & & 0.15 & \multicolumn{2}{|c|}{0.3} & \multicolumn{2}{|c|}{0.8} \\
\hline Int. P X F & & & & & & & & & & & 1 & & & & & \\
\hline C.V. \% (P and F) & \multicolumn{2}{|c|}{7.4} & \multicolumn{2}{|c|}{6.1} & \multicolumn{2}{|c|}{10.08} & \multicolumn{2}{|c|}{11.39} & \multicolumn{2}{|c|}{10.2} & \multicolumn{2}{|c|}{10.6} & & & & \\
\hline
\end{tabular}

Table.2 Dry pod yield, kernel yield, haulm yield and harvest index of groundnut as influenced by different treatments under summer season (Pooled data of three years)

\begin{tabular}{|c|c|c|c|c|c|c|c|c|c|c|c|c|c|c|c|c|}
\hline \multirow[b]{2}{*}{ Treatments } & \multicolumn{4}{|c|}{ Dry pod yield $\left(\mathrm{kg} \mathrm{ha}^{-1}\right)$} & \multicolumn{4}{|c|}{ Kernel yield $\left(\mathrm{kg} \mathrm{ha}^{-1}\right)$} & \multicolumn{4}{|c|}{ Haulm yield $\left(\mathrm{kg} \mathrm{ha}^{-1}\right)$} & \multicolumn{4}{|c|}{ Harvest Index } \\
\hline & $\begin{array}{c}\mathrm{F}_{1}: \mathbf{7 5} \\
\% \\
\text { RDF }\end{array}$ & $\begin{array}{c}\text { F2 : } \\
100 \% \\
\text { RDF }\end{array}$ & $\begin{array}{c}\text { F3 : } \\
125 \% \\
\text { RDF }\end{array}$ & Mean & $\begin{array}{c}\text { F }_{1}: \\
75 \\
\% \\
\text { RDF }\end{array}$ & $\begin{array}{c}\text { F2 : } \\
100 \% \\
\text { RDF }\end{array}$ & $\begin{array}{c}\text { F3 : } \\
125 \% \\
\text { RDF }\end{array}$ & Mean & $\begin{array}{c}\mathrm{F}_{1}: \\
75 \% \\
\mathrm{RDF}\end{array}$ & $\begin{array}{c}\text { F2: } \\
100 \\
\% \\
\text { RDF }\end{array}$ & $\begin{array}{c}\text { F3 : } \\
125 \% \\
\text { RDF }\end{array}$ & Mean & $\begin{array}{c}\mathrm{F}_{1}: \mathbf{7 5} \\
\% \\
\mathrm{RDF}\end{array}$ & $\begin{array}{c}\text { F2 : } \\
100 \% \\
\text { RDF }\end{array}$ & $\begin{array}{l}\text { F3 : } 125 \\
\% \text { RDF }\end{array}$ & Mean \\
\hline$P_{1}:(30 \times 15 \mathrm{~cm})$ & 2367 & 2812 & 3172 & 2784 & 1674 & 2013 & 2302 & 1996 & 2820 & 3268 & 3802 & 3296 & 0.32 & 0.33 & 0.33 & 0.33 \\
\hline$P_{2}:(30 \times 10 \mathrm{~cm})$ & 2689 & 3005 & 3317 & 3004 & 1900 & 2137 & 2377 & 2138 & 3033 & 3371 & 3828 & 3411 & 0.33 & 0.34 & 0.33 & 0.33 \\
\hline$P_{3}:(25 \times 10 \mathrm{~cm})$ & 2810 & 3229 & 3488 & 3175 & 1970 & 2279 & 2470 & 2240 & 3390 & 3728 & 4060 & 3726 & 0.32 & 0.33 & 0.33 & 0.32 \\
\hline$P_{4}(20 \times 10 \mathrm{~cm})$ & 3177 & 3434 & 3635 & 3415 & 2189 & 2394 & 2555 & 2379 & 3598 & 3948 & 4413 & 3986 & 0.32 & 0.32 & 0.32 & 0.32 \\
\hline Mean & 2761 & 3120 & 3403 & 3094 & 1933 & 2206 & 2426 & 2188 & 3210 & 3579 & 4026 & 3605 & 0.32 & 0.33 & 0.33 & 0.33 \\
\hline $\begin{array}{l}\text { Comparing the } \\
\text { means of }\end{array}$ & \multicolumn{2}{|c|}{ S.E. \pm} & \multicolumn{2}{|c|}{ LSD (0.05) } & \multicolumn{2}{|c|}{ S.E. \pm} & \multicolumn{2}{|c|}{ LSD (0.05) } & \multicolumn{2}{|c|}{ S.E. \pm} & \multicolumn{2}{|c|}{$\operatorname{LSD}(0.05)$} & \multicolumn{2}{|c|}{--} & \multicolumn{2}{|c|}{--} \\
\hline $\begin{array}{l}\text { Planting Geometry } \\
\text { (P) }\end{array}$ & \multicolumn{2}{|c|}{50} & \multicolumn{2}{|c|}{148} & \multicolumn{2}{|c|}{33} & \multicolumn{2}{|c|}{99} & \multicolumn{2}{|c|}{64} & \multicolumn{2}{|c|}{189} & \multicolumn{2}{|c|}{--} & \multicolumn{2}{|c|}{--} \\
\hline Fertility levels (F) & \multicolumn{2}{|c|}{33} & \multicolumn{2}{|c|}{95} & \multicolumn{2}{|c|}{24} & \multicolumn{2}{|c|}{69} & \multicolumn{2}{|c|}{51} & \multicolumn{2}{|c|}{146} & \multicolumn{2}{|c|}{--} & \multicolumn{2}{|c|}{--} \\
\hline Int. P X F & \multicolumn{2}{|c|}{67} & \multicolumn{2}{|c|}{190} & \multicolumn{2}{|c|}{49} & \multicolumn{2}{|c|}{139} & \multicolumn{2}{|c|}{103} & \multicolumn{2}{|c|}{293} & & & - & \\
\hline C.V. \% $(\mathrm{P}$ and $\mathrm{F})$ & & & & & & 9 & 6. & & & & 8 & & & & - & \\
\hline
\end{tabular}


Table.3 Economics of groundnut production as influenced by different treatments (Mean data of three years)

\begin{tabular}{|c|c|c|c|c|c|c|c|c|c|c|c|c|c|c|c|c|}
\hline \multirow[b]{2}{*}{ Treatments } & \multicolumn{4}{|c|}{ Cost of cultivation $\left(\mathrm{Rs} \mathrm{ha}^{-1}\right)$} & \multicolumn{4}{|c|}{ Gross returns $\left(\mathrm{Rs} \mathrm{ha}^{-1}\right)$} & \multicolumn{4}{|c|}{ Net returns $\left(\mathrm{Rs} \mathrm{ha}^{-1}\right)$} & \multicolumn{4}{|c|}{ B:C ratio } \\
\hline & $\begin{array}{c}\mathrm{F}_{1}: \mathbf{7 5} \\
\% \\
\mathrm{RDF}\end{array}$ & $\begin{array}{c}\text { F2 : } \\
100 \% \\
\text { RDF }\end{array}$ & $\begin{array}{c}\text { F3 : } \\
125 \% \\
\text { RDF }\end{array}$ & Mean & $\begin{array}{c}F_{1}: 75 \\
\% \\
\text { RDF }\end{array}$ & $\begin{array}{c}\text { F2 : } \\
100 \% \\
\text { RDF }\end{array}$ & $\begin{array}{c}\text { F3 : } \\
125 \% \\
\text { RDF }\end{array}$ & Mean & $\begin{array}{l}\mathrm{F}_{1}: 75 \\
\% \mathrm{RDF}\end{array}$ & $\begin{array}{c}\text { F2 : } \\
100 \% \\
\text { RDF }\end{array}$ & $\begin{array}{c}\text { F3 : } \\
125 \% \\
\text { RDF }\end{array}$ & Mean & $\begin{array}{c}\mathrm{F}_{\mathbf{1}}: \mathbf{7 5} \\
\% \\
\text { RDF }\end{array}$ & $\begin{array}{c}\text { F2 : } \\
100 \% \\
\text { RDF }\end{array}$ & $\begin{array}{c}\text { F3 : } \\
125 \% \\
\text { RDF }\end{array}$ & Mean \\
\hline$P_{1}:(30 \times 15 \mathrm{~cm})$ & 72481 & 79504 & 87119 & 79701 & 124011 & 147139 & 166181 & 145777 & 51530 & 67635 & 79062 & 66076 & 1.71 & 1.85 & 1.91 & 1.82 \\
\hline$P_{2}:(30 \times 10 \mathrm{~cm})$ & 83697 & 90050 & 97248 & 90332 & 140498 & 156976 & 173521 & 156998 & 56801 & 66927 & 76272 & 66667 & 1.68 & 1.74 & 1.78 & 1.74 \\
\hline$P_{3}:(25 \times 10 \mathrm{~cm})$ & 91026 & 99064 & 106190 & 98760 & 147265 & 168886 & 182511 & 166221 & 56239 & 69822 & 76321 & 67461 & 1.62 & 1.70 & 1.72 & 1.68 \\
\hline$P_{4}(20 \times 10 \mathrm{~cm})$ & 103493 & 109421 & 115691 & 109535 & 166029 & 179607 & 190583 & 178740 & 62536 & 70186 & 74892 & 69205 & 1.60 & 1.64 & 1.65 & 1.63 \\
\hline Mean & 87674 & 94510 & 101562 & 94582 & 144451 & 163152 & 178199 & 161934 & 56776 & 68643 & 76637 & 67352 & 1.65 & 1.74 & 1.76 & 1.72 \\
\hline
\end{tabular}

Produce and input rates

\begin{tabular}{|c|c|c|c|c|c|c|}
\hline Rabi Season & $\begin{array}{l}\text { Pod yield } \\
\left(\mathrm{Rs} \mathrm{kg}^{-1}\right)\end{array}$ & $\begin{array}{c}\text { Haulm } \\
\left(\mathrm{Rs} \mathrm{kg}^{-1}\right)\end{array}$ & $\begin{array}{c}\mathrm{N} \\
\left(\mathrm{Rs} \mathrm{kg}^{-1}\right)\end{array}$ & $\begin{array}{c}\mathbf{P}_{2} \mathbf{O}_{5} \\
\left(\mathrm{Rs} \mathrm{Kg}^{-1}\right)\end{array}$ & $\begin{array}{c}\text { FYM } \\
\left(\text { Rs tonne }^{-1}\right)\end{array}$ & $\begin{array}{c}\text { Labour charges } \\
\text { (Rs. day }{ }^{-1} \text { ) }\end{array}$ \\
\hline 2013-14 & 45 & 1.50 & 10.80 & 21.00 & 1000 & 162 \\
\hline $2014-15$ & 45 & 1.50 & 12.26 & 37.50 & 1000 & 180 \\
\hline $2015-16$ & 50 & 2.0 & 12.26 & 37.50 & 1000 & 180 \\
\hline
\end{tabular}

\begin{tabular}{|l|c|}
\hline \multicolumn{1}{|c|}{ Treatments } & Seed rate in pods (kg/ha) \\
\hline $\mathrm{P}_{1}$ : Plant Population @ 2.22 lakhs ha $^{-1}(30 \times 15 \mathrm{~cm})$ & 200 \\
\hline $\mathrm{P}_{2}$ : Plant Population @ 3.33 lakhs ha ${ }^{-1}(30 \times 10 \mathrm{~cm})$ & 300 \\
\hline $\mathrm{P}_{3}$ : Plant Population @ 4.0 lakhs ha ${ }^{-1}(25 \times 10 \mathrm{~cm})$ & 360 \\
\hline $\mathrm{P}_{4}$ : Plant Population @ 5.0 lakhs ha ${ }^{-1}(20 \times 10 \mathrm{~cm})$ & 450 \\
\hline
\end{tabular}




\section{Yield}

The pooled data (Table 2) revealed that groundnut variety, TKG Bold was sown with planting geometry of $20 \times 10 \mathrm{~cm}$ i.e. plant population@5 lakhs ha ${ }^{-1}\left(\mathrm{P}_{4}\right)$ recorded the significantly highest pod, kernel and haulm yield $\left(3415 \mathrm{~kg}, 2379 \mathrm{~kg}\right.$ and $3986 \mathrm{~kg} \mathrm{ha}^{-1}$, respectively) over rest of the planting geometries. However, groundnut sown with planting geometry $\mathrm{P}_{2}$ i.e. plant population @ 3.33 lakhs ha $^{-1}$ (30 X 10) (3004 kg, $2138 \mathrm{~kg}$ and $3411 \mathrm{~kg} \mathrm{ha}^{-1}$, respectively) was at par with $\mathrm{P}_{1}$ planting geometry i.e. plant population@ 2.22 lakhs ha $^{-1}$ (30 X 15) (2784 $\mathrm{kg}, 1996 \mathrm{~kg}$ and $3296 \mathrm{~kg} \mathrm{ha}^{-1}$, respectively) for haulm yield of groundnut. These results are in line as reported by Reddy et al., (2014)

Application of $125 \%$ RDF $\left(\mathrm{F}_{3}\right)$ to groundnut crop recorded significantly highest pod, kernel and haulm yield (3403, 2426 and 4026 $\mathrm{kg} \mathrm{ha}^{-1}$, respectively) over $100 \% \operatorname{RDF}\left(\mathrm{F}_{2}\right)$ (3120, 2206 and $3579 \mathrm{~kg} \mathrm{ha}^{-1}$, respectively) and $75 \% \operatorname{RDF}\left(\mathrm{F}_{1}\right)$ level $(2761,1933$ and $3210 \mathrm{~kg} \mathrm{ha}^{-1}$, respectively). The use of $75 \%$ RDF recorded the least pod, kernel and haulm yield of groundnut. Application of $\mathrm{N}$ and $\mathrm{P}$ fertilizers influences the yield of the crop. Fertilizers promotes vegetative as well as root growth and increases the yield of groundnut (Jakusko and Dakato, 2015). Dadson and Petkov (1976) reported that the high application of nitrogen application is required for high yield of the crop.

\section{Economics}

The maximum net returns of Rs. 69,205/- $\mathrm{ha}^{-1}$ was observed with planting geometry $\mathrm{P}_{4}$ i.e. plant population@5 lakhs ha ${ }^{-1}(20 \times 10 \mathrm{~cm})$, however the highest cost to benefit ratio of 1:1.82 was noticed with plant population@ 2.22 lakhs ha ${ }^{-1}(30 \times 15 \mathrm{~cm})$ i.e. $\mathrm{P}_{1}$. These results are in conformity with findings of Meena (2011)
In case of levels of fertilizers, maximum net monitory returns of Rs. $76,637 /-$ ha $^{-1}$ with higher cost to benefit ratio $(1: 1.76)$ was noticed under use of $125 \%$ RDF fertilizer level (Table 3).

\section{Interaction effect}

Interaction effect between planting geometries and fertility levels was found to be significant in respect of plant height, number of pods plant ${ }^{-1}$ and pod weight plant ${ }^{-1}$ of groundnut. The groundnut variety, TKG Bold sown with planting geometry $\mathrm{P}_{1}$ i.e. $30 \times 15$ $\mathrm{cm}$ (Plant Population of 2.22 lakhs $\mathrm{ha}^{-1}$ ) and fertilized with $125 \%$ RDF level $\left(\mathrm{P}_{1} \mathrm{~F}_{3}\right)$ recoded significantly highest number of pods plant $^{-1}(20.0)$ and pod weight plant ${ }^{-1}(26.6 \mathrm{~g})$ over rest of treatment combinations. Whereas planting geometry $\mathrm{P}_{4}$ i.e. $20 \times 10 \mathrm{~cm}$ (plant population@ 5 lakhs ha ${ }^{-1}$ ) with application of $125 \%$ RDF level $\left(\mathrm{P}_{4} \mathrm{~F}_{3}\right)$ recorded significantly highest plant height (53.7) over rest of treatment combinations.

Similarly, interaction effect between planting geometries and fertility levels was found to be significant in respect of pod, kernel and haulm yield of groundnut. The groundnut cultivar, TKG Bold sown with planting geometry, $\mathrm{P}_{4}$ i.e. $20 \quad \mathrm{X} \quad 10 \mathrm{~cm}$ (plant population@5 lakhs ha ${ }^{-1}$ ) with application of $125 \%$ RDF level $\left(\mathrm{P}_{4} \mathrm{~F}_{3}\right)$ recorded significantly maximum pod, kernel and haulm yield (3635, 2555 and $4413 \mathrm{~kg} \mathrm{ha}^{-1}$, respectively) over all other treatment combinations, but was at par with treatment combination $\left(\mathrm{P}_{3} \mathrm{~F}_{3}\right)$ i.e. groundnut sown by $25 \times 10 \mathrm{~cm}$ planting geometry and fertilized with $125 \% \mathrm{RDF}$, for pod and kernel yield (3488 and $2471 \mathrm{~kg} \mathrm{ha}^{-1}$, respectively) of groundnut.

Groundnut cultivar, TKG Bold was sown with planting geometry, $\mathrm{P}_{4}$ i.e. $20 \mathrm{X} 10 \mathrm{~cm}$ (plant population@ 5 lakhs ha ${ }^{-1}$ ) with application of $125 \%$ RDF level noticed highest pod yield 
(3635 kg ha $\mathrm{kg}^{-1}$ ) of groundnut, which was followed by planting geometry $\mathrm{P}_{3}$ i.e. $25 \mathrm{X} 10$ $\mathrm{cm}\left(3488 \mathrm{~kg} \mathrm{ha}^{-1}\right), \mathrm{P}_{2}$ i.e. 30 X $10 \mathrm{~cm}(3317$ $\left.\mathrm{kg} \mathrm{ha}^{-1}\right)$ and $\mathrm{P}_{1}$ i.e. $30 \times 15 \mathrm{~cm}\left(3172 \mathrm{~kg} \mathrm{ha}^{-1}\right)$ with same fertilizer level i.e.125\% RDF. However, the highest cost to benefit ratio (1:1.91) observed in planting geometry $\mathrm{P}_{1}$ i.e. 30 X $15 \mathrm{~cm}$ with $125 \%$ RDF level.

Hence it was concluded that to obtain higher profitability from rabi summer season, the groundnut cultivar, TKG Bold be sown with planting geometry of $30 \times 15 \mathrm{~cm}$ (plant population @ 2.22 lakhs $\mathrm{ha}^{-1}$ ) with application of $125 \%$ RDF with highest cost to benefit ratio of 1:1.91 under South Konkan Coastal zone of Maharashtra.

\section{References}

Anonymous. 2013. Agriculture at a glance. Directorate of Economics and Statistics, Department of Agriculture and Cooperation, Ministry of Agriculture, Government of India, New Delhi.

Fageria, N.K., Baligar, V.C. and Jones, C. 1997. Growth and mineral nutrition of field crops $2^{\text {nd }}$ Ed. Marcel Dekker, Inc, New York 1001 k, Pp: 494.

Gomez, K.A. and Gomez, A.A. 1983. Statistical Procedure for Agricultural Research Workers. $2^{\text {nd }}$ Edn. An
International Rice Research Institute Book (Jonh Wiley \& Sons eds), Inc. 605 Third Avenue New York, 10158.

Haricharan Reddy, K., Arun kumar, J.S. and Ghosh, G. (2014). Effect of plant spacings on the yield and yield attributes of groundnut varieties. Internat. J. agric. Sci., 10(1): 79-81.

Indiastat, 2016. Seasonwise Area, Production and Productivity of groundnut in India. www.indiastat.com

Jakusko., B.B. and Dakato, M.E. 2015. Effect of NPK fertilizer rates on seed yield of some local cultivars of Bambara groundnut (Vigna subterranean L.). Internat. J. of Chemical, Enviornmental and Biological sci. 3(1): 67-70

Meena, B. 2011. Effect of planting geometry and nitrogen management on groundnut (Arachis hypogaea L.) in loamy soil of Rajasthan, Indian J. of Agric. Sci. 81 (1):1-9

Morshed Alem, A.T.M., Sarker, M.A.R., Mostofa, M.G., Ali, S..M.M and Mollah A.F. 2002. Yield and quality of groundnut (Arachis hypogaea L.) as affected by planting geometry and number of plants per hill. J. of Biological sci. 2(6): 392-394.

Panse, V.G. and Sukhatme, P.V. 1985. Statistical Methods for Agricultural Workers. $4^{\text {th }}$ Ed. ICAR, New Delhi.

\section{How to cite this article:}

Waghmode, B.D., A.S. Kambale, V.C. Navhale, P.D. Chendge and Mahadkar, U.V. 2017. Effect of Plant Population and Fertilizer Doses on Yield Attributes, Yield and Economics of Summer Groundnut. Int.J.Curr.Microbiol.App.Sci. 6(11): 2670-2675. doi: https://doi.org/10.20546/ijcmas.2017.611.314 